\title{
Estudio del bienestar psicológico, emocional y social en la vejez
} Study of well-being psychological, emotional or social in old age

\section{Cortese, Ricardo}

\section{Fernández Canales, María Mercedes}

mercedesfernandez.1987@gmail.com

\section{Siegien, Natalia Berenice}

Universidad Abierta Interamericana. Facultad de Psicología y Relaciones Humanas* DOI: 10.32654/CONCIENCIAEPG.3-1.7

Para referenciar este artículo:

Cortese, R., Fernández Canales, M. M., \& Siegien, N. (2018). Estudio del bienestar psicológico, emocional y social en la vejez. REVISTA ConCiencia EPG, 3(1), 101-115. doi: https://doi.org/10.32654/ CONCIENCIAEPG.3-1.7

\section{Resumen}

Fecha de recepción: 13 -03 - 2018 | Fecha de aceptación: 03-06 -2018

(1)

onsiderando el envejecimiento acelerado y el aumento de la esperanza de vida, se buscó identificar el tipo de bienestar (psicológico, emocional o social) que predominaba en adultos mayores y analizar si existían diferencias significativas entre el grupo de viejos-jóvenes y el de viejos-viejos definidos por Neugarten (1999).

Se realizó un estudio de corte transversal, descriptivocomparativo y se trabajó con una muestra de $\mathrm{N}=218$ participantes de ambos sexos de entre 65 y 98 años de edad de Buenos Aires. Se construyó un cuestionario socio-demográfico ad-hoc y se administró el Mental Health Continuum- Short Form de Keyes (Lupano, De la Iglesia, Castro \& Keyes, 2016).

Los resultados indicaron que tanto en lamuestrageneral como en ambos grupos, el bienestar psicológico fue el predominante. Además, los viejos jóvenes obtuvieron puntuaciones más altas en el bienestar emocional, social y total en comparación con los viejos-viejos.

Palabras clave: Envejecimiento; viejos Jóvenes, viejos viejos, bienestar psicológico, bienestar social bienestar Emocional 


\section{Summary}

onsidering the accelerated aging and the increase in life expectancy, this study searched to identify the type of well-being (psychological, emotional or social) that prevailed in elderly adults and to analyze if there were significant differences between the group of young-elderly people and the group of old-elderly people defined by Neugarten (1999).

For this, a cross-sectional, descriptivecomparative study was carried out, in which we worked with a non-probabilistic sample consisting of $\mathrm{N}=218$ participants of both sexes between 65 and 98 years old living in Buenos Aires.

An ad-hoc socio-demographic questionnaire was constructed and the Keyes Mental Health Continuum-Short Form was administered (Lupano, De la Iglesia, Castro \& Keyes, 2016).

The obtained results indicated that both in the general sample and in both groups, psychological well-being was predominant. In addition, the young-elderly people obtained higher scores in emotional, social and total well-being compared to the old-elderly ones.

Keywords: Aging, young elderly people, old elderly people, psychological well being, social well being, emotional well being

\section{Introducción}

A lo largo de la historia el estudio de la vejez se ha centrado en el deterioro físico 1 y cognitivo que esta etapa evolutiva conlleva, colaborando en relacionarla con déficits y pérdidas. Sin embargo, estudios más recientes han demostrado que las personas mayores poseen elevados niveles de bienestar, satisfacción y fortalezas (Arias, Sabatini \& Soliverez, 2011).

Según la Organización Mundial de la Salud [OMS] (2015), es cierto que el envejecimiento se relaciona con la disminución gradual de las capacidades físicas y mentales, pero no todas las personas envejecen de la misma manera o linealmente de acuerdo a su edad cronológica.

No obstante, diversas investigaciones han demostrado que la felicidad y el bienestar están directamente relacionados con la salud de los ancianos e incluyen que "los programas basados en la Psicología Positiva facilitan una mejor calidad de vida en las personas, previniendo y disminuyendo la aparición de trastornos emocionales" (Ortega, Ramírez \& Chamorro 2015, p. 2).

A su vez, es importante destacar que por primera vez en la actualidad nos encontramos ante la población más longeva de la historia, ya que a nivel mundial la esperanza de vida ha superado los 65 años y se estima que para el año 2020 la población de adultos mayores será superior a la de los niños (OMS, 2015).

El envejecimiento es definido como un conjunto de modificaciones físicas, psicológicas y sociales que suceden a lo largo de toda la vida; desde su concepción hasta la muerte, los seres humanos viven en una permanente interrelación biológica 
y sociocultural en la que el envejecimiento es un proceso continuo (Lombardo \& Krzemien, 2008). Gallardo (2013), retomando los aportes teóricos de Busse (1969), señala que existen dos tipos de envejecimiento, el primario y el secundario.

El primero, es inevitable y se relaciona con el deterioro biológico natural de todos los seres humanos; mientras que en el segundo, el deterioro natural se encuentra agravado por la presencia de alguna enfermedad, hábitos de nutrición, salud y factores ambientales.

Considerando el proceso continuo que define al envejecimiento como parte del ciclo vital, este estudio toma la perspectiva de Lombardo y Krzemien (2008), quienes proponen pensar al desarrollo humano de manera multidimensional, debido a que los cambios que atraviesan las personas durante la vida tienen lugar en el dominio biológico, psicológico y social y de forma multidireccional, ya que suceden cambios que afectan y modifican las conductas.

Asimismo, la literatura científica ha empezado a prestar mayor atención al concepto de envejecimiento activo o exitoso. Los autores Rowe y Kahn (1997) lo definen como una interrelación entre buena salud, capacidad física, cognitiva y compromiso con la vida.

De acuerdo a un estudio realizado por Fernández et al. (2010), el envejecimiento activo abarca un conjunto de factores biológicos, psicológicos y sociales; lo presentan aquellas personas que se perciben satisfechas con la vida, no presentan enfermedades, se autoperciben saludables, indican no necesitar ayuda, poseen un estado mental alto y se incorporan en actividades productivas.
En Argentina, el Ministerio de Salud de la Nación (2017) considera al envejecimiento activo como un proceso mediante el cual se aprovechan las oportunidades para obtener un bienestar físico, psíquico y social, promoviendo la idea de que la edad no debe funcionar como barrera para realizar actividades físicas, culturales y/o sociales que tengan un impacto positivo en la salud y el bienestar.

La vejez es considerada una etapa en la vida del ser humano expresada a través de la edad y marcada por una serie de cambios físicos, psicológicos y sociales (Ramos, Meza, Maldonado, Ortega \& Hernández, 2009). Sin embargo, históricamente ha sido pensada de dos formas, una positiva y otra negativa.

A principios del siglo XX y con la ayuda de la medicina moderna, se comenzó a considerar al anciano como una persona cercana a la muerte, cuyo cuerpo se caracterizaba por la pérdida de vitalidad y energía, comenzando a formar la alianza entre la vejez con términos negativos como invalidez y enfermedad (Iacub, 2011).

Adicionalmente, se sumaron diversos estudios centrados en el deterioro físico y cognitivo que esta etapa evolutiva conlleva, colaborando en asociarla con déficits y pérdidas (Arias et al., 2011).

En pos de eliminar los estereotipos negativos y homogeneizadores, Fernández (2004) sostiene que el desarrollo humano es constante, no finaliza al alcanzar su madurez física, así como tampoco inicia un proceso de deterioro cuando un adulto mayor se 
jubila, pues la vejez es una etapa no muy bien determinada a la cual llegan las personas con una variabilidad de experiencias y aprendizajes que los hacen únicos y diferentes entre sí.

Diversos estudios han evidenciado que algunas de las variables que pueden incrementar el bienestar en las personas son: el trabajo -remunerado o no-, la plasticidad -la capacidad de adaptarse positivamente a los cambios del entorno-, la realización de actividades físicas -deportes, caminar- y productivas -jardinería, compras- (Fernández, 2009).

Pese a esto, aún en la actualidad persisten prejuicios, prácticas y políticas que sostienen creencias negativas acerca de la vejez y que obstaculizan el tipo de atención que se presta a las personas mayores privándolas muchas veces de actividades sociales y económicas (OMS, 2016).

A la vez, los avances en el área médica y social permiten y facilitan el incremento de la duración del ciclo vital (Sánchez, 2000).

La gerontóloga social Neugarten (1999) agrupa a la población adulta mayor en viejosjóvenes y viejos-viejos. Dentro del grupo de viejos-jóvenes, se encuentran aquellas personas que tienen entre 65 a 74 años de edad, pudiendo alcanzar en algunos casos los 79 años y se caracterizan por tener salud óptima, fortaleza física, psíquica y disfrutar de un relativo bienestar económico.

Además, incluye personas bien integradas en sus familias y comunidades que tienen un alto grado de educación $\mathrm{y}$ desean permanecer activos realizando distintas actividades.

En el grupo de viejos-viejos, se encuentran los adultos mayores de 80 años de edad y se caracterizan por ser personas que, a causa de su deterioro mental o físico, requieren asistencia sanitaria y social.

Con respecto a esta clasificación, es importante aclarar que Neugarten (1999) considera a esta división por grupos etarios, como una alternativa para ordenar la enorme diversidad existente entre los adultos mayores, pero no es una agrupación cronológica.

Los términos viejos-jóvenes y viejos-viejos solo refieren al estado de salud mental y física y a la participación de los sujetos en el ámbito familiar y comunitario.

Por otra parte, se refleja que durante mucho tiempo la Psicología focalizó sus estudios en los aspectos negativos de la naturaleza humana e incluso en patologías como la depresión y la ansiedad, dejando casi de lado los temas referentes a la salud y al bienestar. La aparición de la Psicología Positiva vino a cambiar esta visión tradicional, aportando nuevas perspectivas (Alarcón, 2009).

De esta manera, se comenzó a estudiar el bienestar y si bien al principio no existía un acuerdo entre los autores sobre una definición científica, varias disciplinas y campos de la psicología fueron relacionándolo con la calidad de vida, la salud mental y con características personales (Ballesteros, Medina \& Caycedo, 2006).

El estudio del bienestar en el área de psicología, toma las reflexiones filosóficas que pueden encontrarse en los escritos de Platón, 
Aristóteles, Confucio y Séneca relacionada con términos tales como vida buena, vida virtuosa y el cuidado del alma (Rangel \& Alonso, 2010).

Ryan y Deci (2001) explican que la investigación acerca del bienestar gira en torno a dos visiones contrapuestas: una denominada hedonista y otra llamada eudaimonista.

La visión hedonista equipara al bienestar con la felicidad, la experimentación del placer corporal, la persecución de los propios intereses y el alcance de logros, mientras que la eudaimonista separa al bienestar de la felicidad y sostiene que se alcanza cuando los sujetos viven de acuerdo a su verdadero yo y realizan actividades que son congruentes con sus valores.

En esta misma línea, el estudio del bienestar se puede determinar en dos dimensiones. La primera, denominada bienestar emocional o subjetivo que se encuentra relacionada con el estudio de las emociones positivas y con la evaluación cognitiva que hacen los sujetos de su satisfacción con la vida (Diener, Suh, Lucas \& Smith, 1999).

La segunda, es multidimensional y contempla los aspectos sociales y del entorno de un sujeto para evaluar su bienestar psicológico, es decir, se concentran en los aspectos relacionales (Ryff, 1989; Keyes, 1998; Ryff \& Singer, 2008).

Desde el surgimiento de la Psicología Positiva, ha nacido un gran interés en prestar atención a las fortalezas personales y cómo contribuyen al bienestar. Se han comenzado a desarrollar técnicas que, en lugar de eliminar emociones negativas, estimulen la aparición de emociones positivas (Vera, 2006).
Como ya se ha mencionado, se han desplegado dos líneas de investigación muy marcadas, la eudaimónica y la hedónica; de hecho, en la actualidad existe un acuerdo entre los investigadores que afirman que el bienestar puede ser abordado de manera global, teniendo en cuenta ambos enfoques: una vida que es rica en ambas actividades (hedónicas y eudaimónicas) puede contribuir al más alto nivel de bienestar (Huta \& Ryan, 2010).

En este contexto, una de las contribuciones más importante para la psicología positiva fue la aparición del concepto florecimiento humano, que describe a un sujeto con un estado de salud mental y un funcionamiento humano óptimos (Momtaz, Hamid, Haron \& Bagat, 2016).

Según Keyes (2005), el florecimiento refleja altos niveles de bienestar emocional, psicológico y social. En el desarrollo de este concepto el autor unificó las perspectivas hedónicas y eudaimónicas, creando un instrumento que evalúa los aspectos sociales, emocionales y psicológicos del bienestar.

El presente estudio utiliza el Modelo del Continuo de Salud Mental [MCSM] creado por Keyes (2002), el cual parte de la concepción de que la salud mental positiva y la enfermedad mental están relacionadas pero sus dimensiones son diferentes, ya que una serie continua indica la presencia o ausencia de salud y otra la presencia o ausencia de enfermedad.

Por consiguiente, Keyes (2002; 2005; 2007) enfatiza que para evaluar el grado de salud mental positiva deben tenerse en cuenta los 
tres tipos de bienestar, es decir el emocional, el social y el psicológico.

El bienestar emocional se relaciona con las emociones positivas y la satisfacción con la vida. El bienestar psicológico tiene que ver con el propio agrado, responder eficientemente a responsabilidades cotidianas $\mathrm{y}$ tener relaciones positivas con otros.

Por último, el bienestar social refiere al tipo de funcionamiento de un individuo con su entorno, implica tener algo para contribuir a la sociedad, sentirse parte de la misma y estar en sintonía con la forma en la que funciona la sociedad (Keyes \& Shapiro, 2004; Keyes, 2006).

En función de lo expuesto, este estudio se propuso como objetivo principal identificar el tipo de bienestar: social, psicológico, emocional que predomina en adultos mayores e indagar si existen diferencias significativas entre dos grupos de edad, los viejos-jóvenes: 65 a 79 años de edad y los viejos-viejos: 80 años en adelante.

Dentro del estudio tradicional del bienestar, hay evidencia de que los niveles de bienestar subjetivo aumentan con la edad y existen niveles más altos de afecto positivo y satisfacción en las personas casadas que en las solteras (Lacey et al., 2006; Moyano \& Ramos, 2007; Vivaldi \& Barra, 2012).

En Argentina, Gerstenbluth, Rossi y Triunfo (2008) hallaron que los adultos tienen un $70 \%$ de probabilidad de estar satisfechos con la vida y que la felicidad decrece con la edad hasta los 54 años y luego comienza a incrementarse.

También, Arias et al. (2009) exploraron posibles variaciones en el bienestar psicológico en dos grupos de adultos mayores argentinos de entre 60 a 75 y 76 a 91 años de edad y no hallaron diferencias significativas entre ambos grupos.

Posteriormente, el equipo liderado por Arias (2011) llevó a cabo un taller con un grupo de adultos mayores residentes de la ciudad de Mar del Plata, donde trabajaron los aspectos positivos en la vejez y demostraron que las personas mayores poseen elevados niveles de bienestar psicológico y satisfacción con la vida.

En lo que sigue, se mencionan los estudios donde se ha utilizado el Mental Health Continuum Short Form [MHC-SF] y se consideran relevantes para la presente investigación.

Uno de los estudios que empleó el MHCSF fue el de Westerhof y Keyes (2010), para evaluar la enfermedad y la salud mental en adultos holandeses de entre 18 y 87 años de edad. A partir de ello, encontraron que los adultos mayores puntuaron con niveles más altos de bienestar emocional y más bajos de bienestar psicológico que los adultos jóvenes. Otro, fue realizado en Estados Unidos, por Snowden, Dhingra, Keyes y Anderson (2010) que, por medio de un diseño longitudinal, compararon los cambios del bienestar de adultos, dividiéndolos en 3 cohortes de edad: 45 y 54 años, 55-64 años y 65-74 años.

Los resultados mostraron que los participantes mayores experimentaron un ligero descenso del bienestar en comparación con los más jóvenes.

Finalmente, Góngora y Castro (2017) realizaron el primer estudio en América Latina que evaluó el estado de salud mental según la clasificación del modelo propuesto por Keyes en 
una muestra de adultos argentinos de entre 18 y 85 años de edad (edad media 40,62 años).

Los resultados reflejaron que la mayoría de la muestra se clasificó con salud mental moderada $(54,9 \%)$, es decir, con niveles medios de bienestar, seguida de floreciente $(39,7 \%)$, es decir con altos niveles de bienestar, y sólo una minoría se clasificó como languideciente $(5,4 \%)$, es decir con bajos niveles de bienestar.

En conclusión, exceptuando el último estudio mencionado, que sólo es una aproximación a la temática de este trabajo, no se evidencian investigaciones en la población argentina que identifiquen el tipo de bienestar que predomina en la vejez utilizando modelos que integren las concepciones tradicionales del bienestar -hedónica y eudaimónica-.

Por lo tanto, el presente estudio toma en cuenta el Modelo del Continuo de Salud Mental de Keyes (2002) con el propósito de identificar cuál es el aspecto del bienestar que privilegian los adultos mayores de Capital Federal y Gran Buenos Aires.

\section{Método}

Participantes: Se utilizó una muestra no probabilística (Hernández et al., 2006) conformada por $\mathrm{N}=218$ participantes (mujeres $63,8 \%$ - hombres $36,2 \%$ ) de entre 65 y 98 años de edad (edad media 76,81; desvío típico 7,87) que asistían a centros de jubilados de la Ciudad Autónoma de Buenos Aires y del Gran Buenos Aires, con la siguiente distribución:

- Grupo de Viejos Jóvenes, es decir adultos que tienen entre 65 y 79 años de edad (Neugarten, 1999) al momento de realizar la entrevista, compuesto por $\mathrm{N}=110$ participantes (74 mujeres -36 hombres, edad media 70 años; desvío típico $3,98)$.

- Grupo de Viejos Viejos, es decir adultos que tienen de 80 años de edad en adelante (Neugarten, 1999) al momento de realizar la entrevista, compuesto por $\mathrm{N}=108$ participantes ( 65 mujeres - 43 hombres, edad media 83,75 años; desvío típico 3,64).

Se excluyeron los casos en que los participantes presentasen patologías graves; que se encuentren institucionalizados o bajo el cuidado de un tercero, ya sea familiar y/o profesional.

Instrumentos: en este estudio se utilizaron dos instrumentos como: Cuestionario sociodemográfico ad hoc, se construyó un cuestionario breve, para los fines exclusivos de lainvestigaciónque incluyó preguntas cerradas con opción de respuesta única y múltiple, que se centró en conseguir los siguientes datos: sexo, edad, lugar de residencia y presencia de alguna enfermedad mental o física que afecte el grado de autonomía del participante.

Escala Mental Health Continuum- Short Form [MHC-SF] de Keyes (2006, adaptación Argentina de Lupano, De la Iglesia, Castro $\&$ Keyes, 2017). Es un cuestionario de autoreporte que consta de 14 ítems que miden el grado de bienestar emocional (3 ítems), psicológico (6 ítems) y social (5 ítems) en el que se pide al examinado que valore sus sentimientos del mes pasado en una escala de 5 puntos $(0=$ nunca, $1=$ una vez o dos veces al mes, $2=$ una vez a la semana, $3=$ dos o tres veces a la semana, $4=$ casi todos los días, 5= todos los días). 
Para la evaluación se deben sumar los ítems correspondientes a cada escala y se calcula una media por cada dimensión. Debe arrojar un valor entre 0 y 5 puntos. No posee ítems inversos. A mayor puntaje mayor nivel de bienestar en cada una de las dimensiones consideradas.

En la adaptación realizada en la población argentina la consistencia interna fue 0,89 para el MHC-SF y se ha confirmado la estructura de tres factores de la escala y buena validez convergente: $\mathrm{a}=0,86$ para el bienestar emocional, $\mathrm{a}=0,86$ para el bienestar psicológico y a $=0,75$ para el bienestar social.

Procedimiento: En primer lugar, se solicitó la autorización al Presidente de cada centro de jubilados y se explicó el objetivo del trabajo a realizar con los socios en los momentos donde finalizaban las diferentes actividades.

Luego, se entregó a los participantes un protocolo que incluía: una reseña del presente estudio; la solicitud del consentimiento informado, en el que se informaba que la participación era voluntaria y anónima y que los datos recogidos eran de carácter confidencial y los instrumentos, que brindaban una descripción que contenía y explicaba la consigna.

Los instrumentos fueron completados por los participantes de manera individual y autónoma, brindándose la posibilidad de que ante alguna duda podían dirigirse al investigador que se encontraba en una sala aparte. A su vez, con la intención de cumplir con el anonimato de las respuestas, se procedió a identificar a cada participante con un número de protocolo y la variable nombre se reemplazó por él.

Por último, para el análisis y procesamiento de los datos se utilizó el programa informático estadístico SPSS versión 20.0 en español.

\section{Resultados}

En primer lugar se llevó a cabo el análisis estadístico descriptivo de las tres dimensiones del bienestar que evalúa el MHC-SF (emocional, social, psicológico), para calcular la puntuación media de cada dimensión en la muestra general y en los dos grupos de adultos mayores, es decir, viejos-jóvenes y viejos-viejos.

Posteriormente, se realizó el análisis de normalidad en las variables de estudio por medio de la prueba de Shapiro-Wilk para realizar el análisis de diferencias de grupos. A continuación, se presentan los resultados estadísticos obtenidos.

El análisis estadístico descriptivo, expuesto en la Tabla 1, evidenció que en la muestra general, conformada por $\mathrm{N}=218$ participantes, predominó una puntuación mayor de bienestar psicológico, seguida por la del bienestar emocional y una baja puntuación de bienestar social.

En el grupo de Viejos Jóvenes, compuesto por $\mathrm{N}=110$ participantes, se reportó un puntaje mayor de bienestar emocional y bienestar psicológico, mientras que un bajo puntaje de bienestar social - ver Tabla 2.

Por último, en el grupo de Viejos-Viejos, compuesto por $\mathrm{N}=108$ participantes, predominó una puntuación mayor de bienestar psicológico, seguida por la del bienestar emocional y una baja puntuación de bienestar social - ver Tabla 3. 
Artículos Empíricos

Tabla 1

Niveles de bienestar en la muestra general

\begin{tabular}{lccccc}
\multicolumn{1}{c}{$N=218$} & Media & Mediana & DT & Mín. & Máx. \\
\hline $\begin{array}{l}\text { Bienestar } \\
\text { emocional }\end{array}$ & 3.4 & 3.6 & 1.1 & 0 & 5 \\
$\begin{array}{l}\text { Bienestar } \\
\text { social }\end{array}$ & 2.1 & 2 & 1.2 & 0 & 5 \\
$\begin{array}{l}\text { Bienestar } \\
\text { psicológico }\end{array}$ & 3.5 & 3.8 & 1 & 0 & 5 \\
Bienestar total & 3 & 3 & 1 & 0 & 5 \\
\hline
\end{tabular}

Tabla 2

Niveles de bienestar en el grupo de Viejos Jóvenes

\begin{tabular}{lccccc}
\hline \multicolumn{1}{c}{$N=110$} & Media & Mediana & DT & Mín. & Máx. \\
\hline $\begin{array}{l}\text { Bienestar } \\
\text { emocional }\end{array}$ & 3.6 & 4 & 1 & 0 & 5 \\
$\begin{array}{l}\text { Bienestar } \\
\text { social }\end{array}$ & 2.3 & 2.6 & 1 & 0 & 5 \\
$\begin{array}{l}\text { Bienestar } \\
\text { psicológico }\end{array}$ & 3.6 & 3.8 & 1 & 0 & 5 \\
Bienestar total & 3.1 & 3.2 & 1 & 0 & 5 \\
\hline
\end{tabular}

Tabla 3

Niveles de bienestar en el grupo de Viejos Viejos

\begin{tabular}{lccccc}
\hline \multicolumn{1}{c}{$N=108$} & Media & Mediana & DT & Mín. & Máx. \\
\hline $\begin{array}{l}\text { Bienestar } \\
\text { emocional }\end{array}$ & 3.2 & 3.3 & 1.2 & 0 & 5 \\
$\begin{array}{l}\text { Bienestar } \\
\text { social }\end{array}$ & 1.9 & 1.8 & 1.1 & 0 & 5 \\
$\begin{array}{l}\text { Bienestar } \\
\text { psicológico }\end{array}$ & 3.4 & 3.7 & 1.1 & 0 & 5 \\
$\begin{array}{l}\text { Bienestar } \\
\text { total }\end{array}$ & 2.8 & 3 & 1 & 0 & 5 \\
\hline
\end{tabular}


Por otra parte, se realizó la Prueba de normalidad, análisis de Shapiro-Wilk; los valores obtenidos mostraron que las variables analizadas no poseen una distribución normal en la muestra general.

En consecuencia, se decidió utilizar estadística no paramétrica para el análisis de diferencia de grupos, evaluado mediante el test U de Mann Whittney - ver Tabla 4.
En el análisis de diferencias de grupo según la clasificación de Neugarten (1999), se observaron diferencias estadísticamente significativas en los niveles de bienestar emocional $(p=.03)$, social $(p=.00)$ y total $(p=.02)$ entre ambos grupos de adultos mayores, pues los Viejos Jóvenes presentaron mayores niveles en los tres tipos de bienestar que los Viejos Viejos - ver Tabla 5.

\begin{tabular}{lccc} 
Tabla 4 \\
Análisis de normalidad en la Muestra \\
\multicolumn{4}{c}{$\begin{array}{c}\text { Shapiro-Wilk } \\
\text { Estadístico }\end{array}$} \\
\hline &, 93 & 218 & gl \\
\hline $\begin{array}{l}\text { Bienestar } \\
\text { emocional }\end{array}$ &, 97 & 218 &, 00 \\
$\begin{array}{l}\text { Bienestar social } \\
\text { Bienestar } \\
\text { psicológico } \\
\text { Bienestar total }\end{array}$ &, 90 & 218 &, 00 \\
\hline
\end{tabular}

Tabla 5

Diferencias grupales según la clasificación Neugarten

\begin{tabular}{lll}
\hline & Clasificación Neugarten & $\mathrm{p}$. \\
\hline $\begin{array}{l}\text { Bienestar } \\
\text { emocional }\end{array}$ & $\begin{array}{l}\text { Viejos jóvenes }=(\mathrm{R}=118.20) \\
\text { Viejos viejos }=(\mathrm{R}=100.63)\end{array}$ & .03 \\
$\begin{array}{l}\text { Bienestar } \\
\text { social }\end{array}$ & $\begin{array}{l}\text { Viejos jóvenes }=(\mathrm{R}=121.54) \\
\text { Viejos viejos }=(\mathrm{R}=97.24)\end{array}$ & .00 \\
$\begin{array}{l}\text { Bienestar } \\
\text { psicológico }\end{array}$ & $\begin{array}{l}\text { Viejos jóvenes }=(\mathrm{R}=113.20) \\
\text { Viejos viejos }=(\mathrm{R}=105.74)\end{array}$ \\
Bienestar total & $\begin{array}{l}\text { Viejos jóvenes }=(\mathrm{R}=119.07) \\
\text { Viejos viejos }=(\mathrm{R}=99.75)\end{array}$ \\
\hline
\end{tabular}

Nota: Prueba U de Mann Whitney. 


\section{Discusión}

El presente estudio fue motivado por el interés de conocer qué tipo de bienestar predominaba en los adultos mayores argentinos por medio del Modelo del Continuo de Salud Mental [MCSM] creado por Keyes (2002), con el fin de aportar información pertinente para la elaboración de nuevos modelos teóricos que contemplen el nivel de bienestar obtenido, el diseño de nuevas estrategias de abordaje y la generación de nuevas intervenciones basadas en la Psicología Positiva, que favorezcan el estado de bienestar en la vejez.

Además, se buscó indagar si existían diferencias significativas entre dos grupos de edad basados en la clasificación de la gerontóloga social Neugarten (1999), el grupo de ViejosJóvenes y el de Viejos-Viejos, para generar propuestas orientadas a mejorar su calidad de vida y adaptabilidad y para comprender nuevas formas de integración psicofísica de estos grupos etarios.

Teniendo en cuenta los resultados obtenidos en la muestra general, predominó la dimensión del bienestar psicológico; a partir de esto, se deduce que durante la vejez la persona experimenta su propio agrado, respondiendo a sus responsabilidades diarias y fomentando las relaciones positivas con otros (Keyes, 2006).

Además, considerando la pequeña diferencia con el nivel de bienestar emocional, tanto en la muestra general como en los dos grupos etarios, puede pensarse que los participantes privilegian la vivencia de emociones positivas y se encuentran satisfechos con su vida.

Sin embargo, fue significativa la baja puntuación obtenida en la dimensión de bienestar social, que persistió a lo largo del análisis, puesto que ésta refiere a las creencias que tiene el mayor acerca de las circunstancias en las que vive junto al funcionamiento dentro del entorno social (Keyes \& Shapiro, 2004). Por ello, se cree importante considerar estos resultados para repensar políticas de integración social dentro de este grupo etario.

Por otra parte, al comparar los resultados entre los dos grupos, se encontró que los Viejos Jóvenes presentaron mayores niveles de bienestar emocional, social y total que los Viejos Viejos. Tomando los aportes realizados por Jiménez et al. (2016) y Ortega et al. (2015) podrían pensarse para el grupo de los viejos-viejos, dispositivos y actividades que incrementen el afecto positivo, la satisfacción con la vida y la integración social, para generar el impacto positivo en la salud y el bienestar propio de la concepción actual del envejecimiento activo (Ministerio de Salud de la Nación, 2017; Fernández, et. al, 2010).

Considerando los resultados de este trabajo, se encontró similitud con los del estudio de Snowden et al. (2010) que demostraron que los adultos mayores estadounidenses experimentaban un ligero descenso en el bienestar total en comparación con los participantes más jóvenes.

Sin embargo, no coincidieron con el estudio de Westerhof y Keyes (2010), ya que los autores hallaron que los adultos mayores holandeses puntuaron con niveles más altos de bienestar emocional; tampoco, con la exploración de Arias y Soliverez (2009) que no hallaron diferencias significativas en los niveles de bienestar en dos grupos de edad; ni 
con el estudio de Lacey et al. (2006), en el que se halló un aumento en los niveles de felicidad con la edad y, por último, con la investigación de Moyano y Ramos (2007) que encontraron que las personas mayores presentaron un mayor nivel de bienestar subjetivo.

De igual modo, estas diferencias podrían ser explicadas por la cultura, el alcance de los objetivos de los estudios, el sesgo de la edad y el modelo teórico junto al instrumento de evaluación empleados.

En suma, aquí solo se expuso una aproximación al estudio de las dimensiones del bienestar (emocional, psicológico y social) en la vejez por medio del Modelo del Continuo de Salud Mental, en el que Keyes (2002) unificó las concepciones teóricas tradicionales del bienestar -hedónica y eudaimónica-.

Por esta razón, se espera que futuras líneas de investigación tengan en cuenta seguir indagando en esta temática y controlar las limitaciones presentes en este estudio, es decir, el control de las características sociodemográficas que no se han considerado para el análisis (ej. estado civil, nivel de educación, situación económica, condiciones de vida, etc.) y además, se debe tener en cuenta que al haberse empleado un muestreo no probabilístico los resultados de este estudio no pueden ser generalizados a toda la población de adultos mayores argentinos.

\section{Conclusión}

En conclusión, el objetivo principal de este estudio fue identificar qué tipo de bienestar (social, psicológico, emocional) predominaba en adultos mayores e indagar si existían diferencias significativas entre dos grupos de edad, los viejosjóvenes: 65 a 79 años de edad y los viejos-viejos: 80 años en adelante.

Los resultados descriptivos evidenciaron que en los adultos mayores $(\mathrm{N}=218)$ que participaron en este estudio, predominó un mayor puntaje en la dimensión de bienestar psicológico, seguido por la del bienestar emocional y un puntaje menor de bienestar social.

Teniendo en cuenta la división de la muestra general según la clasificación de Neugaraten (1999), se encontró que en el grupo de Viejos Jóvenes $(\mathrm{N}=110)$ predominó el bienestar emocional, seguido por el bienestar psicológico y, por último, el bienestar social, mientras que en el grupo de Viejos-Viejos ( $\mathrm{N}=108)$ se presentó un puntaje mayor de bienestar psicológico, seguido por el bienestar emocional y una baja puntuación de bienestar social.

Por otra parte, el análisis de diferencia de grupos, reveló que el grupo de Viejos Jóvenes presentó mayores niveles de bienestar emocional, social y total que el de los Viejos-Viejos. 


\section{Referencias}

Alarcón, R. (2009). Psicología de la felicidad. Introducción a la psicología positiva. Revista de Psicología, 28(2), 411-414.

Arias, C. \& Soliverez, C. (2009). El Bienestar Psicológico en la vejez. ¿Existen diferencias por grupos de edad? II Congreso Internacional de Investigación de la Facultad de Psicología. Universidad Nacional de La Plata.

Arias, C., Sabatini, M. \& Soliverez, V. (2011). El trabajo con aspectos positivos para la promoción de la salud en la vejez. III Congreso Internacional de Investigación y Práctica Profesional en Psicología XVIII Jornadas de Investigación Séptimo Encuentro de Investigadores en Psicología del MERCOSUR. Facultad de Psicología Universidad de Buenos Aires, Buenos Aires.

Ballesteros, B., Medina, A. \& Caycedo, C. (2006). El bienestar psicológico definido por asistentes a un servicio de consulta psicológica en Bogotá, Colombia. Univ. Psychol. Bogotá, 5(2), 239-258.

Busse, E. (1969). Theories of aging. En E.W. Busse y E. Pfeiffer (Eds.), Behavior and adaptation in late life (pp. 11-32). Boston: Little Brown.

Castro, A. (2009). El bienestar psicológico: cuatro décadas de progreso. Revista Interuniversitaria de Formación del Profesorado, 23(3), 43-72.

Castro, A. (2010). Fundamentos de psicología positiva. Buenos Aires: Paidós.
Castro, A. \& Grinhauz, A. (2011). Las rutas de acceso al bienestar, relaciones entre bienestar hedónico eudaemónico. III Congreso Internacional de Investigación y Práctica Profesional en Psicología XVIII Jornadas de Investigación Séptimo Encuentro de Investigadores en Psicología del MERCOSUR. Facultad de Psicología - Universidad de Buenos Aires, Buenos Aires.

Diener, E. \& Suh, E. (1998). Age and subjective well-being: An international analysis. Annual Review of Gerontology and Geriatrics, 17, 304-324.

Diener, E., Suh, E., Lucas, R. \& Smith, H. (1999). Subjective well-being: Three decades of progress. Psychological Bulletin, 125(2), 276-302.

Fernández, R. (2004). Psicología de la vejez. Humanitas, 1, 27-38.

Fernández, R. (2009). Jubilación y Salud. Humanitas, 37.

Fernández, R., García, M., Zamarrón, M., López, M., Molina, J., Díez, N., Montero, P. \& Schettini, R. (2010). Envejecimiento con éxito: criterios y predictores. Psicothema, 22(4), 641647.

Gallardo,L.(2013).Apoyosocialyetnicidaden personas mayores de la región de Arica y Parinacota-Chile: una propuesta para la intervención en trabajo social (Tesis Doctoral). Universidad Complutense de Madrid, España. 
Gerstenbluth, M., Rossi, M. \& Triunfo, P. (2008). Felicidad y salud: una aproximación al bienestar en el Río de la Plata. Estudios de Economía, 35(1), 65-78.

Góngora, V. \& Castro, A. (2017). Assessment of the Mental Health Continuum in a Sample of Argentinean Adults. Psychology, 8, 303-318.

Huta, V., \& Ryan, R. (2010). Pursuing pleasure or virtue: The differential and overlapping well-being benefits of hedonic and eudaimonic motives. Journal of Happiness Studies, 11(6), 735-762.

Iacub, R. (2011). Erótica y Vejez Perspectivas de occidente. $2^{a}$ ed. Argentina: Paidós.

Iacub, R. (2011). Identidad y envejecimiento. Buenos Aires: Paidós.

Jiménez, M., Izal, M. \& Montorio, I. (2016). Programa para la mejora del bienestar de las personas mayores. Estudio piloto basado en la psicología positiva. Suma psicológica, 23, 51-59.

Keyes, C. (1998). Social well-being. Social Psychology Quarterly, 61, 121-140.

Keyes, C. (2005). Mental Illness and/or Mental Health? Investigating Axioms of the Complete State Model of Health. Journal of Consulting and Clinical Psychology, 73(3), 539-548.
Keyes, C. (2006). Mental Health in Adolescence: Is America's Youth Flourishing? American Journal of Orthopsychiatry, 76(3), 395-402.

Keyes, C. (2007). Promoting and protecting mental health as flourishing: A complementary strategy for improving national mental health. American Psychologist, 62(2), 95108.

Lacey, H., Smith, D. \& Ubel, P. (2006). Hope I die before I get old: Mispredicting happiness across the adult lifespan. Journal of Happiness Studies, 7(2), 167-182.

Lupano, M., De la Iglesia, G., Castro, A. \& Keyes, C. (2016). The Mental Health ContinuumShort Form (MHC-SF) in the Argentinean Context: Confirmatory Factor Analysis and Measurement Invariance. Europe's Journal of Psychology, 13(1), 93-108.

Ministerio de Salud (2017). Envejecimiento activo. Recuperado de http://www.msal. gob.ar/index.php/component/content/ article/48-temas-de-salud-de-la-a-a-laz/389-envejecimiento-activo.

Momtaz, Y., Hamid, T., Haron, S., \& Bagat, M. (2016). Flourishing in later life. Archives of gerontology and geriatrics, 63, 85-91.

Moyano, E. \& Ramos, N. (2007). Bienestar subjetivo: midiendo satisfacción vital, felicidad y salud en población chilena de la Región Maule. Revista Universum, 22(2), 177-193.

Organización Mundial de la Salud (2015). Envejecimiento y Salud. Recuperado de: 
http://www.who.int/mediacentre/factsheets/ fs $404 / \mathrm{es} /$

Organización Mundial de la Salud (2016). Las actitudes negativas acerca del envejecimiento y la discriminación contra las personas mayores pueden afectar su salud. Recuperado de: http://www.who. int/mediacentre/news/releases/2016/ discrimination-ageing-youth/es/

Ortega, A. Ramírez, E. \& Chamorro, A. (2015). Una intervención para aumentar el bienestar de los mayores. European Journal of Investigation in Health, Psychology and Education, 5(1), 23-33.

Ramos, J., Meza, A., Maldonado, I., Ortega, M. \& Hernández, M. (2009). Aportes para una conceptualización de la vejez. Revista de Educación y Desarrollo, 11, 47-56.

Rangel, J. \& Alonso, L. (2010). El estudio del bienestar psicológico subjetivo. Una breve revisión teórica. Artículos arbitrados, 14, $265-275$.

Rowe, J. \& Kahn, R. (1997). Successful Aging. The Cerontologist, 37(4), 433-440.

Ryan, R. \& Deci, E. (2001). On happiness and human potentials: A review of research on hedonic and eudaimonic well-being. Annual Review of Psychology, 52(1) 141-166.

Ryff, C. (1989). Happiness is everything, or is it? Explorations on the meaning of psychological well-being. Journal of personality and social psychology, 57(6), 1069-1081.
Ryff, C. (1995). Psychological well-being in adult life. Current Directions in Psychological Science, 4, 99-104.

Ryff, C. \& Singer, B. (2008). Know thyself and become what you are: A eudaimonic approach to psychological well-being. Journal of Happiness Studies, 9, 13-39.

Sánchez Salgado, C. (2000). Gerontología social. Buenos Aires: Editorial Espacio.

Seligman, M. (2014). Florecer. La nueva psicología positiva y la búsqueda del bienestar. México: Océano.

Seligman, M. \& Csikszentmihalyi, M. (2000). Positive Psychology: An Introduction. American Psychologist, 55(1), 5-14.

Soliverez, C. (2015). Bienestar: un recorrido teórico $\mathrm{y}$ empírico hacia resultados positivos mayores. Revista Kairós Gerontología, 18(20), 15-34.

Vera, B. (2006). Psicología positiva: una nueva forma de entender la psicología. Papeles del Psicólogo, 27(1), 3-8.

Vivaldi, F. \& Barra, E. (2012). Bienestar Psicológico, Apoyo Social Percibido y Percepción de Salud en Adultos Mayores. Terapia psicológica, 30(2), 23-29.

Westerhof, G. \& Keyes, C. (2010). Mental illness and mental health: The two continua model across the lifespan. Journal of adult development, 17(2), 110-119. 